\title{
The association between imitation recognition and socio-communicative competencies in chimpanzees ( Pan troglodytes)
}

\author{
Sarah M. Pope ${ }^{1,2}$, Jamie L. Russell ${ }^{1,3}$ and William D. Hopkins ${ }^{1,3} *$ \\ ${ }^{1}$ Neuroscience Institute and Language Research Center, Georgia State University, Atlanta, GA, USA \\ ${ }^{2}$ Laboratoire de Psychologie Cognitive, Aix-Marseille Université, Marseille, France \\ ${ }^{3}$ Division of Developmental and Cognitive Neuroscience, Yerkes National Primate Research Center, Atlanta, GA, USA
}

Edited by:

Thomas Bugnyar, Universität Wien, Austria

\section{Reviewed by:}

Rachel Louise Kendal, The University of Durham, UK

Elsa Addessi, National Research

Council of Italy, Italy

Tomas Persson, Lund University,

Sweden

\section{*Correspondence:}

William D. Hopkins, Neuroscience Institute and Language Research Center, Georgia State University, Atlanta, GA 30302, USA

e-mail:whopkins4@gsu.edu;

whopkin@emory.edu
Imitation recognition provides a viable platform from which advanced social cognitive skills may develop. Despite evidence that non-human primates are capable of imitation recognition, how this ability is related to social cognitive skills is unknown. In this study, we compared imitation recognition performance, as indicated by the production of testing behaviors, with performance on a series of tasks that assess social and physical cognition in 49 chimpanzees. In the initial analyses, we found that males were more responsive than females to being imitated and engaged in significantly greater behavior repetitions and testing sequences. We also found that subjects who consistently recognized being imitated performed better on social but not physical cognitive tasks, as measured by the Primate Cognitive Test Battery. These findings suggest that the neural constructs underlying imitation recognition are likely associated with or among those underlying more general socio-communicative abilities in chimpanzees. Implications regarding how imitation recognition may facilitate other social cognitive processes, such as mirror self-recognition, are discussed.

Keywords: imitation recognition, mirror self recognition, social cognition, chimpanzees, imitation

\section{INTRODUCTION}

An important form of human cognition and learning is imitation. Imitation is defined as reproducing an action after seeing it performed (modified from Thorndike, 1898). It has been suggested by some that imitative learning is a uniquely human form of learning and this claim has stimulated a significant body of research in non-human animals, and particularly non-human primates (Hayes and Hayes, 1952; Tomasello et al., 1987, 1993; Custance et al., 1995; Voelkl and Huber, 2000; Myowa-Yamakoshi et al., 2004; Call et al., 2005; Horner and Whiten, 2005; Tennie et al., 2006; Bard, 2007; Buttelmann et al., 2007; Hobaiter and Byrne, 2010). Further, the neural constructs underlying imitation appear to be rooted in a proposed action-recognition system of mirror neurons that encode both the somatosensory input of another individual performing an action as well as the motor output required for the individual to perform the action themselves. This mirror neuron system (MNS), in essence, allows for the deciphering of the actions, goals, and mental states of a social partner by mapping them onto familiar counterparts that express the same motor repertoires (see Iacoboni, 2009 for review).

Beyond imitation, as an action-understanding mechanism, the MNS has been hypothesized to reflect a 'like-me' recognition of other humans as sentient beings (Meltzoff, 2002; Meltzoff and Prinz, 2002). Thus, the MNS has been hypothesized to underlie other social cognitive processes that are predicated on the "likeme" system such as empathy, theory of mind, sympathy, and joint attention (Meltzoff and Decety, 2003; Decety, 2010). In support of this hypothesis are cross-sectional and longitudinal developmental data from children showing significant associations between imitation skills and a variety of other socio-communicative abilities such as empathy (Chartrand and Bargh, 1999), joint attention (Carpenter et al., 1995; Charman et al., 2000), expressive language (Tomasello et al., 1993; Slaughter and McConnell, 2003) and, of special interest, mirror self-recognition (MSR; Asendorpf et al., 1996; Nielsen and Dissanayake, 2004). The adaptive significance of recognizing oneself in a mirror is difficult to imagine. However, if we consider that MSR is dependent on mapping the visual input of one's own actions onto motor output and imitation is dependent on mapping the visual input of another's actions onto motor output, their association is logical. Given the functional activation of the MNS during MSR in humans, (Uddin et al., 2005, 2007), it is not implausible that the MNS may have evolved to serve imitative and social cognitive purposes with MSR abilities arising as a byproduct.

The MNS's involvement in social exchanges is not unidirectional: the same mechanisms that allow us to reproduce another individual's actions (i.e., imitation production) presumably underlie the ability to recognize when we are being imitated by another individual (herein referred to as imitation recognition; Decety et al., 2002; Nadel, 2002). Imitation recognition is divided into two categories: implicit recognition, whereby the individual being imitated simply directs their gaze toward an imitative experimenter rather than a non-imitative experimenter and explicit recognition, whereby the individual being imitated employs atypical behaviors to assess the actions of the imitator. For example, testing behaviors are defined as unexpected and 
sudden behaviors performed by the subject while gazing at the imitator (Asendorpf et al., 1996; Meltzoff, 2002; Nielsen et al., 2006). These aptly named behaviors are produced as a means of testing the contingency between the imitator and the imitatee, much like contingency actions often described in children (24 month olds) and apes when looking into a mirror (Gallup, 1970; De Veer and Van Den Bos, 1999; Bard et al., 2006). Implicit imitation recognition has been reported in rhesus (Paukner et al., 2005) and capuchin monkeys (Paukner et al., 2009) while both implicit and explicit imitation recognition has been reported in human children (implicit: 9 month olds; explicit: 14 month olds, 18 month olds) and great apes (Meltzoff, 1990; Asendorpf et al., 1996; Agnetta and Rochat, 2004; Nielsen et al., 2005; Haun and Call, 2008). Explicit testing behaviors employed by humans and apes but not monkeys suggest that advanced imitation recognition capacities may be a derived trait (Gallup, 1977; Bates and Byrne, 2010). Further, a recent cortical connectivity study comparing monkey, chimpanzee, and human MNSs suggests that species differences in imitative abilities may have a neurological foundation based on homologous but differential white matter connections between MNS regions in each species (Hecht et al., 2013).

Despite the heuristic value of the MNS when considered within the context of comparative studies on imitation, the collective findings appear somewhat paradoxical. For instance, mirror neurons were first discovered in macaque monkeys (see Rizzolatti and Luppino, 2001; Rizzolatti and Craighero, 2004), a species for which there is little if any evidence of imitation (but see Ferrari et al., 2006, 2009). With the exception of neonatal imitation in macaques and chimpanzees (Myowa-Yamakoshi et al., 2004; Ferrari et al., 2006; Bard, 2007), evidence of true imitation, such as that demonstrated in "do-as-I-do" types of tasks are rare in the non-human literature (Hayes and Hayes, 1952; Moore, 1992; Custance et al., 1995; Topal et al., 2006; Abramson et al., 2013). Indeed, though a number of studies have demonstrated that non-human primates can learn to solve certain problem-solving tasks by observation, whether these skills are acquired by imitation or other related processes such as emulation and social facilitation remains a topic of considerable debate (Tomasello et al., 1987; Myowa-Yamakoshi and Matsuzawa, 1999; Voelkl and Huber, 2000; Call et al., 2005; Horner and Whiten, 2005; Buttelmann et al., 2007; Carpenter and Call, 2009; Hobaiter and Byrne, 2010). However, the importance of imitation and imitation recognition in primate social interactions becomes apparent when we consider their probable roles in understanding the actions and intentions of others.

The focus of the current study was twofold. First, we sought to replicate and extend previous studies on imitation recognition in chimpanzees by testing a larger sample of individuals as a means of assessing individual differences in performance. Previous studies have tested rather small samples of subjects [between 1 and 11 apes from three different genera Nielsen etal. (2005), Haun and Call (2008)] and here we sought to test a larger cohort within a single ape genus in order to better assess individual differences and the role that sex might have on performance. The second goal was to examine the potential association between imitation recognition and individual differences in social and non-social cognition. A number of studies in typically and atypically developing children have shown that individual differences in social cognitive processes, like play and joint attention, are associated with performance on imitation tasks (Carpenter et al., 1995, 2002; Asendorpf et al., 1996; Charman et al., 2000; Rogers et al., 2003; Nielsen and Dissanayake, 2004; Ingersoll and Schreibman, 2006; Whalen et al., 2006; Hobson and Hobson, 2007). It has been well documented that chimpanzees, and other great apes, engage in some aspects of joint attention and related socio-communicative skills (Leavens et al., 2008; Leavens and Racine, 2009; Leavens, 2012); however, unlike studies in developing children, to what extent imitation performance might be associated with socio-communicative abilities is unknown. While a recent study indicated that infant rhesus macaques produce more affiliative behaviors when an experimenter is imitating them than when producing repetitive behaviors (Sclafani et al., 2014), the relationship between social and imitative abilities in primates is largely unexplored. Here, we initially tested chimpanzees on an imitation recognition task and characterized each individual as performing well or poorly based on how consistently they produced testing behaviors in response to being imitated. We subsequently compared these groups on their previously collected Primate Cognition Test Battery (PCTB) performance. The PCTB is a series of tasks that has been previously used to assess social and non-social cognition in humans, apes, and monkeys (Herrmann et al., 2007, 2010a,b; Russell et al., 2011; Schmitt et al., 2011; Hopkins et al., 2014b). Among these, the PCTB was used to demonstrate differential factor structures underlying the cognitive processes of chimpanzees and human children (2 years of age; Herrmann et al., 2010b) as well as the heritability of cognition in chimpanzees (Hopkins et al., 2014b). We predicted that if imitation recognition is associated with other socio-communicative abilities in chimpanzees, rather than a distinct process, then subjects that perform well on the imitation recognition tasks will perform significantly better on socially oriented PCTB tasks than apes that performed poorly.

\section{MATERIALS AND METHODS SUBJECTS}

Subjects were 16 male and 33 female chimpanzees housed in social groups ranging from 2 to 12 individuals (with the exception of one singly housed male) at the Yerkes National Primate Research Center. Group sizes remained highly consistent between imitation recognition and PCTB test dates, only two subjects' group sizes changed and these were only by +1 and -2 . The subjects ranged in age from 15 to 44 years of age. All of the chimpanzees have been part of a series of behavioral and cognitive studies (Leavens and Hopkins, 1998; Russell et al., 2011; Lyn et al., 2013; Hopkins et al., 2014a; Latzman et al., 2014) but had not been previously tested for imitation recognition prior to this study. All behavioral tests were approved by the local Institutional Animal Care and Use Committee and complied with the Institute of Medicine recommendations for ethical use of chimpanzees in research.

\section{IMITATION RECOGNITION METHODS}

Imitation recognition testing took place between October, 2008 and May, 2010. Subjects voluntarily separated from their social 
groups to participate in each brief test session. Each subject participated in three separate test sessions or blocks that consisted of four 3-min trials. The study was designed so that within a test block, there were two imitation trials (IMs) and two control trials either in an ABBA or BAAB order. In IM trials, the experimenter imitated the subjects' actions as accurately as possible. Following the methods used by Nielsen et al. (2005), we employed three different types of control trials: (1) contingent non-matching (CNM), in which the experimenter produced non-similar actions (i.e., different body part and movement) in response to subjects' actions, (2) non-contingent non-matching (NCNM), in which the experimenter performed a series of preconceived actions at a pre-set pace, and (3) no action (NA), in which the experimenter did not perform any actions at all. Each subject was assigned to one of three preset block orders. The same condition (i.e., $\mathrm{CNM}, \mathrm{CNM}$ ) was used for both control trials within a block. In this way, trial presentation order was cross-balanced across subjects.

\section{IMITATION RECOGNITION ANALYSIS}

Each session was videotaped and all behaviors of interest were coded and analyzed for the presence of testing behaviors using playback on a Sony HDV 1080i Digital HD Videocassette Recorder. Behaviors of interest were defined as any behavior that is directed at an object (i.e., cage banging), is directed at the experimenter (i.e., lip pouting at the experimenter), is an unusual self-directed behavior (i.e., patting head) or is a demonstration of body contingency testing (i.e., running back and forth). Common self-directed behaviors such as scratching, general locomotive behaviors such as shifting positions, and looking behaviors were not included. As indicators of imitation recognition, we were specifically interested in the frequency of three classes of behavioral responses including testing sequences (TS), behavior repetitions (BRs), and testing poses (TPs).

Testing sequences were defined (modified from Asendorpf et al., 1996) as a sequence, lasting for a minimum of $10 \mathrm{~s}$, of at least four successive, different behaviors with no more than $5 \mathrm{~s}$ between any two successive behaviors. For example, one subject exhibited a 30 s sequence consisting of: pulling on the mesh five times, pausing for $4 \mathrm{~s}$, hitting the mesh with their hand two times, hitting the mesh with their foot four times, performing a handstand, hitting the mesh with their foot three times, performing a quick $180^{\circ}$ turn, and then hitting the mesh with their foot 5 times.

Behavior Repetitions were defined as the subject performing the same type of action at least four times with no more than $3 \mathrm{~s}$ between any two repetitions. For example, a subject hit the wall 16 times consecutively. For both TS and BR, the subject had to be looking (directing head and gaze) at the experimenter at the beginning of and continually monitoring the experimenter throughout the sequence.

Testing Poses were defined as the subject looking at the experimenter while holding an atypical body posture (i.e., open mouth, head stand) for more than $3 \mathrm{~s}$. For example, a subject held onto the mesh and open mouth stared at the EXP for $6 \mathrm{~s}$. The number of TS, BR, and TP responses in each experimental condition (IM, $\mathrm{CNM}, \mathrm{NCNM}$, and NA) was the dependent measure of interest.
Inter-rater reliability was assessed for $10.9 \%$ of trials $(n=64)$. Significant Pearson's correlations were calculated for TSs $(r=0.797, p<0.001)$, BRs $(r=0.886, p<0.001)$, and TPs $(r=0.916, p<0.001)$.

\section{PCTB METHODS}

The same subjects tested for imitation recognition were also tested using a modified version of the PCTB as described in Russell etal. (2011) and Hopkins etal. (2014b). PCTB testing took place from January 2008 through June 2009. Briefly, subjects were administered 12 tasks designed to broadly assess social and physical cognition. A brief description of each task is presented in Table 1 (see Russell etal., 2011 for full descriptions). Within the PCTB task, eight tasks assessed physical cognition and included spatial memory, object permanence, rotation, transposition, quantity discrimination, causalityvisual, causality-noise, and tool properties. Four tasks assessed social cognition by requiring subjects to respond to or produce social cues included pointing to cups, comprehension of pointing, attention state, and point/gaze following. Experimenters were blind to the results of the imitation recognition task.

\section{DATA ANALYSIS}

IM tests were performed within each condition (IM vs. CNM, IM vs. NCNM, IM vs. NA). Therefore, in order to test for differences in TS, BR, and TP across the four conditions, we calculated the average number of TS, BR, and TP for all IM conditions and used this as the sole measure. This was done to minimize the number of tests performed on the data and guard against Type I error. Separate mixed model analyses of variance were performed for the TS, BR, and TP behavioral responses. For each analysis, condition was the repeated measure (IM, CNM, NCM, NA) while sex was the between group factor. Alpha was set to $p<0.05$ and all post hoc tests were performed using either Tukey's Honestly Significant Difference or pairwise LSD tests. For the PCTB test, the percentage of correct responses was computed based on performance across trials.

\section{RESULTS IMITATION RECOGNITION RESULTS}

For TSs $F(3,141)=19.70, p=0.001$, BRs $F(3,141)=11.91$, $p=0.001$, and TPs $F(3,141)=13.89, p=0.001$, significant differences were found between the experimental and control conditions. The mean number of TSs, BRs, and TPs for each condition are shown in Figure 1. For all three types of BRs, post hoc analysis indicated that the number of responses in the IM condition were significantly higher than in the CNM, NCNM, and NA conditions (all ps < 0.05). We found no significant differences in the frequency in TSs, BRs, and TPs between the CNM, NCNM, and NA conditions. We also found significant sex differences in the frequency of TSs $F(1,47)=3.93, p=0.05$ (Mean Male $=0.975$, $\mathrm{SE}=0.201$ vs. Mean Female $=0.482, \mathrm{SE}=0.147)$ and in the frequency of BRs $F(1,47)=4.62, p=0.037$ (Mean Male $=2.02, \mathrm{SE}=0.415$ vs. Mean Female $=0.917, \mathrm{SE}=0.302$ ) with males having higher frequencies than females. 
Table 1 | PCTB task descriptions.

\begin{tabular}{|c|c|}
\hline \multicolumn{2}{|l|}{ Physical cognition tasks } \\
\hline Spatial memory (three trials) & $\begin{array}{l}\text { Assessed subjects' ability to remember the locations of two food rewards out of three possible locations. Success } \\
\text { was achieved by only looking in the two correct locations. }\end{array}$ \\
\hline Object permanence (nine trials) & $\begin{array}{l}\text { Assessed subjects' ability to follow a hidden food as it underwent either a single or double displacement; thus, } \\
\text { either one or two of a possible three cups were manipulated. Success was achieved by locating the hidden food } \\
\text { without searching in the location that was not manipulated. }\end{array}$ \\
\hline Rotation (nine Trials) & $\begin{array}{l}\text { Assessed subjects' ability to relocate a hidden food item from among three options following a rotation of all three } \\
\text { options on the horizontal plane. }\end{array}$ \\
\hline Transposition (nine trials) & $\begin{array}{l}\text { Assessed subjects' ability to track and select a food reward that is hidden in one of three locations. The baited } \\
\text { location is then switched with the unbaited locations in one of three ways. }\end{array}$ \\
\hline Relative numbers (13 trials) & Assessed subjects' ability to differentiate between and chose the larger of two quantities of food. \\
\hline Causality noise (six trials) & $\begin{array}{l}\text { Assessed subjects' ability to differentiate between a baited metal container and an unbaited metal container based } \\
\text { on the sound produced when they were shaken. Success was achieved if they chose the baited container. }\end{array}$ \\
\hline Causality visual (six trials) & $\begin{array}{l}\text { Assessed subjects' ability to identify which of two boards and which of two cloths were baited. In each trial type, } \\
\text { the food caused a visible difference in the baited board or cloth. }\end{array}$ \\
\hline Tool properties (six trials) & $\begin{array}{l}\text { Assessed subjects' ability to pull the a functionally correct, baited piece of paper as opposed to one that was not } \\
\text { baited or one that was cut into two pieces. }\end{array}$ \\
\hline \multicolumn{2}{|l|}{ Social cognition tasks } \\
\hline Comprehension (six trials) & $\begin{array}{l}\text { Assessed subjects' ability to use an experimenter's gaze or gaze combined with manual point to identify which of } \\
\text { two objects to touch. }\end{array}$ \\
\hline Production (four trials) & $\begin{array}{l}\text { Assessed subjects' ability to communicate to an experimenter which location had been baited by another } \\
\text { experimenter. }\end{array}$ \\
\hline Attentional state (eight trials) & $\begin{array}{l}\text { Assessed subjects' ability to perform communicative signals in the appropriate modality for the experimenter's } \\
\text { gaze; visual if gaze was directed toward the subject and auditory if it was not. }\end{array}$ \\
\hline Gaze following (three trials) & Assessed subjects' ability to follow the experimenter's upward gaze. \\
\hline
\end{tabular}

\section{IMITATION RECOGNITION AND PCTB RESULTS}

To test for the association between imitation recognition and PCTB performance, we classified the chimpanzees as consistent $(\mathrm{CON})$ or inconsistent (INCON) in their imitation recognition based on the frequency of their TS, BR, and TP responses across the three test conditions. Recall that each chimpanzee received two IM trials within each of the three blocks. Thus, if chimpanzees performed a TS, BR, or TP in two or three of the blocks they were classified as CON. Chimpanzees that produced a TS, BR, or TP in only one or none of tests were classified as INCON. Using this criterion, for TS, there were 24 INCON and 25 CON individuals while there were 22 INCON and $27 \mathrm{CON}$ individuals for the BR behavior. For TP, there were 30 INCON and 19 CON chimpanzees.

Based on each subject's imitation recognition performance for each class of behaviors indicative of imitation recognition, we compared their performance on the PCTB. CON with the original dichotic characterization of PCTB performance (Table 1), we computed summary performance scores based on the social and physical cognition tasks. Thus, the performance on the gaze, attention state, production, and comprehension tasks were averaged together to create a global "social" cognition score. Similarly, the performance scores on the spatial cognition, object permanence, rotation, transposition, relative numbers, causality noise, causality visual, and tool properties were averaged together to create a global "physical" cognition score. These social and physical global cognition scores served as the repeated measure while imitation performance classification (CON, INCON) and sex (Male, Female) served as between group factors. For TSs $F(1,45)=7.37$, $p=0.009$, a significant two-way interaction was found between imitation recognition classification and PCTB performance. The mean social and physical performance scores in chimpanzees classified as CON or INCON based on their TS production is shown in Figure 2. No significant interactions were found between social and physical PCTB scores and BRs or TPs.

Next, to understand the association between imitation recognition measures and individual PCTB task scores, we performed a task-specific analysis with subjects' performance in each РCTB task serving as the repeated measure while imitation performance classification (CON, INCON) and sex (Male, Female) served as between group factors. For the TS $F(11,495)=2.75$, $p=0.002$ and BR $F(11,495)=2.31, p=0.009$ behaviors, significant two-way interactions were found between imitation recognition classification and PCTB performance. No other significant main effects or interactions were found. Post hoc analysis indicated that for chimpanzees classified as $\mathrm{CON}$ on the imitation recognition task, as manifest by either TSs and/or BRs, performed better on the gesture production, comprehension, and attention state measures compared to those classified as INCON 

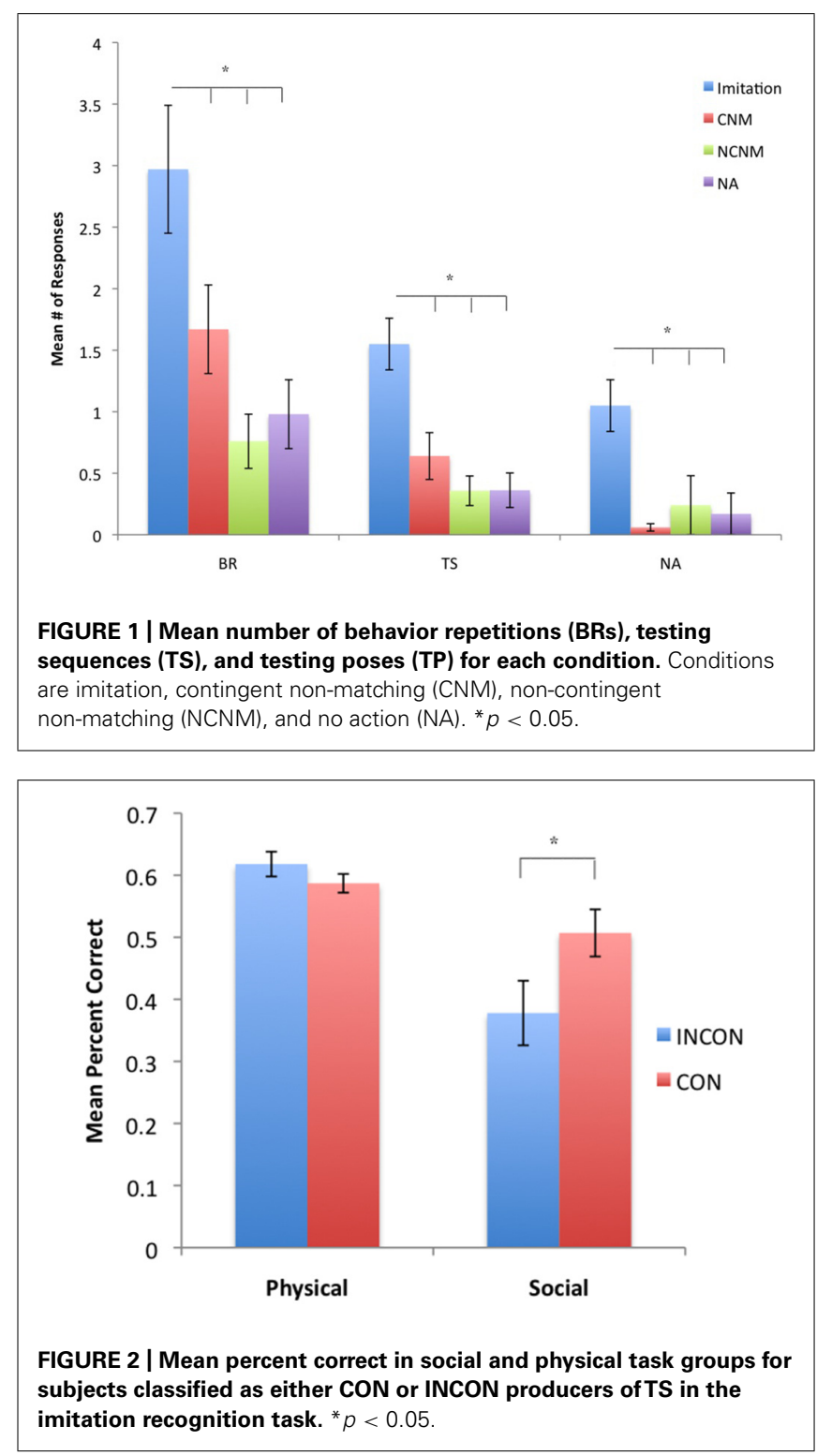

(all ps $<0.05$ ). Performance between the two groups on the remaining tasks was not significantly different from each other. For TPs, there was no significant main effect or interactions with performance on the PCTB tasks. The mean performance score on each PCTB task in chimpanzees classified as CON or INCON based on their TS or BR production are shown in Figures 3 and $\mathbf{4}$, respectively.

\section{DISCUSSION FINDINGS}

The present study investigated the relationship between imitation recognition performance and social and physical cognition in chimpanzees and the results are fairly straight-forward. There were two main findings. First, sex differences were evident in imitation recognition with males showing a higher proclivity to respond to being imitated than females. Second, we found that chimpanzees who performed consistently well on two out of three measures of imitation recognition also had significantly higher scores on three out of the four PCTB social cognition tasks than those that performed inconsistently. Performance in non-social cognitive tasks on the PCTB was not associated with imitation recognition performance. These results support our hypothesis that imitation recognition skills are associated with other aspects of socio-communicative abilities in chimpanzees.

Although our results show that imitation recognition and communicative abilities in chimpanzees are related, the nature of this relationship is unclear. Further research should address how practice in social cognitive tasks might influence imitation recognition abilities, or vice versa. We would further note that defining and quantifying imitation recognition was quite challenging. Though we established inter-rater reliability in scoring TSs, BRs, and TPs, this was challenging and does not lend itself to simple interpretations of the chimpanzees' behavior. Finally, this study used existing PCTB data (Russell et al., 2011; Hopkins et al., 2014b) to measure social and physical cognition but other cognitive measures might have been more sensitive.

\section{ADAPTIVE SIGNIFICANCE OF IMITATION RECOGNITION}

In humans, imitation recognition is suggested to facilitate the interpretation of others' actions and the understanding of others as cognizant beings via the MNS (Meltzoff, 2002; Meltzoff and Prinz, 2002; Iacoboni, 2009). We suggest that imitation recognition may function similarly, in chimpanzee social cognition, affording imitation recognizers with a potentially better understanding of the actions (and intentions?) of others. However, our results only provide support for the association between imitation recognition and communicative skills in chimpanzees. Whether or not the former facilitates the latter is unknown. Further, while it is tempting to suggest that the behavioral homologies between human and chimpanzee imitation systems might reflect homologies within their respective MNSs, future investigation is necessary.

As has been suggested (Asendorpf and Baudonniere, 1993; Mitchell, 1993, 1997; Nielsen and Dissanayake, 2004), we would concur that imitation recognition may be a critical skill that underlies the well-documented abilities of chimpanzees and other great apes to recognize themselves in mirrors (MSR). When initially confronting a mirror, many chimpanzees engage in contingent actions in front of the mirror (Gallup, 1970; Lin et al., 1992; Povinelli et al., 1997), a behavior that looks quite similar to the TSs or BRs that chimpanzees engage in while being imitated. In truth, the most precise imitator is a mirror. Further, it is of note that only apes evince MSR and show explicit imitation recognition (Haun and Call, 2008; Anderson and Gallup, 2011); thus, heuristically, there is the potential that MSR may be closely related to imitation recognition but this warrants further investigation.

We did not anticipate sex differences and it is unclear why males would be more likely to engage in imitation recognition than females. Nonetheless, in some wild chimpanzee populations, it has been reported that males are more gregarious and have stronger association indices than females (Goodall, 1986; Wrangham, 1986; Mitani et al., 2000). It might be argued that responding to being 


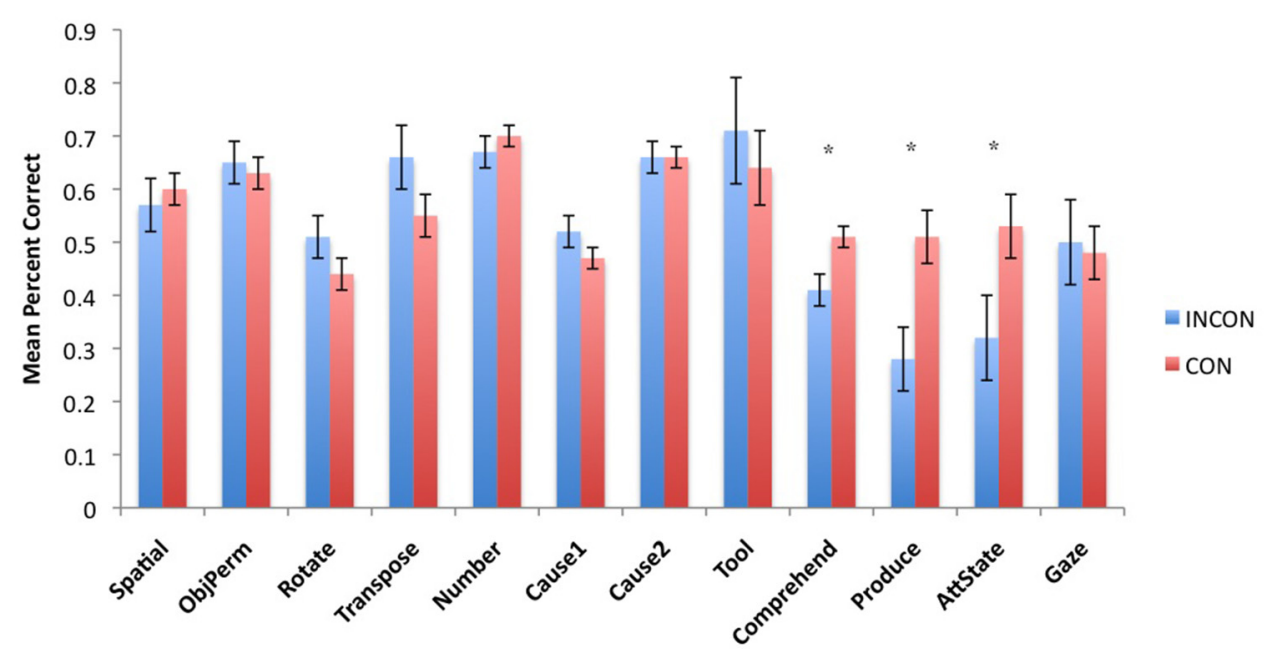

FIGURE 3 | Mean percent correct in each of the 12 PCTB tasks for subjects classified as either CON or INCON producers of TSs in the imitation recognition task. ${ }^{*} p<0.05$.

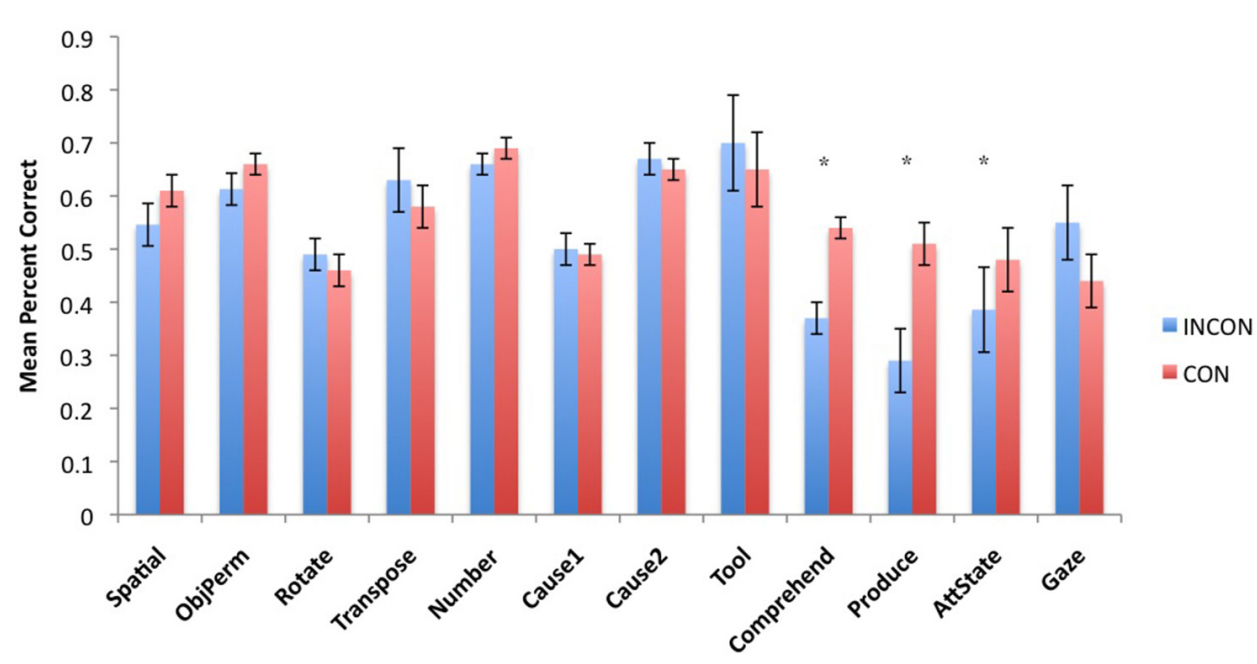

FIGURE 4 | Mean percent correct in each of the 12 PCTB tasks for subjects classified as either CON or INCON producers of BRs in the imitation recognition task. ${ }^{*} p<0.05$.

imitated by engaging in BRs and TSs may reflect this enhanced gregariousness or alternatively an inherent motivation to react to unusual social interactions. Note, the experimenters were female, which conceivably could have lead to increased motivation to participate for male subjects. However, we think this is an unlikely explanation as the same two experimenters conducted the PCTB testing (and many other cognitive and interactive tests Russell et al., 2011; Hopkins and Taglialatela, 2013) in which no sex differences were found. It could also be suggested that male chimpanzees are simply more attentive and reactive to socio-communicative behaviors and this has some advantage in terms of their development and maintenance of social relationships. For instance, Hopkins et al. (2012) and Latzman et al. (2014) have recently found sex dependent influences of a vasopressin receptor gene (AVPR1A) on personality ratings in chimpanzees. Males with a duplication copy of the RS3 coding regions within the AVPR1A were rated as more dominant than those without a copy. It has also been reported that males with the RS3 copy of the AVPR1A gene performed significantly better than males without a copy on a task assessing receptive joint attention, a simple measure of social cognition (Hopkins et al., 2014a). It is intriguing to speculate that males with the RS3 copy might be simply more prosocial and this potentially manifests itself within the context of MSR or related abilities such as imitation recognition. Although our sample was small, when we examined the mean number of TSs, BRs, or TPs in imitation conditions, we found that RS3+ males $($ Mean $=2.52$, $\mathrm{SE}=0.69)$ showed a higher number of responses than RS3+ females $($ Mean $=0.802, \mathrm{SE}=0.448)$, RS3 - males $($ Mean $=2.39$, 
$\mathrm{SE}=55)$, and RS3 - females (Mean $=1.57, \mathrm{SE}=0.42)$. These differences were not significant but the patterns of results were in the right direction and perhaps with a larger sample, these results might reveal some significant genetic effects on imitation recognition in chimpanzees.

\section{CONCLUSION}

The importance of imitative capacities in the human social environment is apparent. By providing evidence for this same association in non-human primates, the current study sets the stage for elucidating the origins of the advanced imitative and social communication found in humans. We argue that the presence of imitation skills in non-human primates may have been driven by the role of the MNS in understanding others as sentient beings. Furthermore, we highlight the likely correspondence between the evolution of imitation and MSR abilities (Sowden and Shah, 2014). We propose that the discovery of a link between imitation and social skills in chimpanzees could suggest that a common neural substrate for these abilities may have been present in our most common ancestor.

\section{ACKNOWLEDGMENTS}

This research was supported in part by NIH grants NS-42867, NS73134, HD-60563, and HD-56232 to WDH. The Yerkes Center is fully accredited by the American Association for Accreditation of Laboratory Animal Care. We are incredibly grateful to Jennifer Schaeffer for her large role in both PCTB and imitation recognition data collection. SMP is funded by Georgia State University's 2CI Primate Social Cognition, Evolution, and Behavior Doctoral Fellowship. American Psychological Association guidelines for the ethical treatment of animals were adhered to during all aspects of this study.

\section{REFERENCES}

Abramson, J. Z., Hernandez-Lloreda, V., Call, J., and Colmenares, F. (2013). Experimental evidence for action imitation in killer whales (Orcinus orca). Anim. Cogn. 16, 11-22. doi: 10.1007/s10071-012-0546-2

Agnetta, B., and Rochat, P. (2004). Imitative games by 9-, 14-, and 18-month-old infants. Infancy 6, 1-36. doi: 10.1207/s15327078in0601_1

Anderson, J. R., and Gallup, G. G. Jr. (2011). Which primates recognize themselves in mirrors? PLoS Biol. 9:e1001024. doi: 10.1371/journal.pbio.1001024

Asendorpf, J. B., and Baudonniere, P. M. (1993). Self-awareness and otherawareness: mirror self-recognition and synchronic imitaiton among unfamilair peers. Dev. Psychol. 29, 88-95. doi: 10.1037/0012-1649.29.1.88

Asendorpf, J. B., Warkentin, V., and Baudonniere, P. M. (1996). Self-awareness and other-awareness. II. Mirror self-recognition, social contingency awareness and synchronic imitation. Dev. Psychol. 32, 313-321. doi: 10.1037/0012-1649.32.2.313

Bard, K. A. (2007). Neonatal imitation in chimpanzees (Pan troglodytes) tested with two paradigms. Anim. Cogn. 10, 233-242. doi: 10.1007/s10071-006-0062-3

Bard, K. A., Todd, B. K., Bernier, C., Love, J., and Leavens, D. A. (2006). Selfawareness in human and chimpanzee infants: what is measured and what is meant by the mark and mirror test? Infancy 9, 191-219. doi: 10.1207/s15327078in0902_6

Bates, L. A., and Byrne, R. W. (2010). Imitation: what animal imitation tells us about animal cognition. Wiley Interdiscip. Rev. Cogn. Sci. 1, 685-695. doi: $10.1002 /$ wcs.77

Buttelmann, D., Carpenter, M., Call, J., and Tomasello, M. (2007). Enculturated chimpanzees imitate rationally. Dev. Sci. 10, 31-33. doi: 10.1111/j.14677687.2007.00630.x

Call, J., Carpenter, M., and Tomasello, M. (2005). Copying results and copying actions in the process of social learning: chimpanzees (Pan troglodytes) and human children (Homo sapiens). Anim. Cogn. 8, 151-163. doi: 10.1007/s10071-0040237-8
Carpenter, M., and Call, J. (2009). Comparing the imitative skills of children and nonhuman apes. Rev. Primatol. 1. doi: 10.4000/primatologie.263

Carpenter, M., Pennington, B. F., and Rogers, S. J. (2002). Interrelations among social-cognitive skills in young children with autism. J. Autism. Dev. Disord. 32, 91-106. doi: 10.1023/A:1014836521114

Carpenter, M., Tomasello, M., and Savage-Rumbaugh, S. (1995). Joint attention and imitative learning in children, chimpanzees, and enculturated chimpanzees. Soc. Dev. 4, 217-237. doi: 10.1111/j.1467-9507.1995.tb00063.x

Charman, T., Baron-Cohen, S., Swettenham, J., Baird, G., Cox, A., and Drew, A. (2000). Testing joint attention, imitation and play as infancy precursors to language and theory of mind. Cogn. Dev. 15, 481-498. doi: 10.1016/S08852014(01)00037-5

Chartrand, T. L., and Bargh, J. A. (1999). The chameleon effect: the perceptionbehavior link and social interaction. J. Pers. Soc. Psychol. 76, 893-910. doi: 10.1037/0022-3514.76.6.893

Custance, D., Whiten, A., and Bard, K. (1995). Can young chimpanzees (Pan troglodytes) imitate arbitrary actions? Hayes and Hayes (1952) revisited. Behaviour 132, 837-850. doi: 10.1163/156853995X00036

De Veer, M. W., and Van Den Bos, R. (1999). A critical review of methodology and interpretation of mirror self-recognition research in nonhuman primates. Anim. Behav. 58, 459-468. doi: 10.1006/anbe.1999.1166

Decety, J. (2010). The neurodevelopment of empathy in humans. Dev. Neurosci. 32, 257-267. doi: 10.1159/000317771

Decety, J., Chaminade, T., Grezes, J., and Meltzoff, A. N. (2002). A PET exploration of neural mechanisms involved in reciprocal imitation. NeuroImage 15, 265-272. doi: 10.1006/nimg.2001.0938

Ferrari, P. F., Paukner, A., Ruggiero, A., Darcey, L., Unbehagen, S., and Suomi, S. J. (2009). Interindividual differences in neonatal imitation and the development of action chains in rhesus macaques. Child Dev. 80, 1057-1068. doi: 10.1111/j.14678624.2009.01316.x

Ferrari, P. F., Visalberghi, E., Fogassi, L., Ruggiero, A., and Suomi, S. J. (2006). Neonatal imitation in rhesus macaques. PLoS Biol. 4:e302. doi: 10.1371/journal.pbio.0040302

Gallup, G. G. (1970). Chimpanzees: self-recognition. Science 167, 86-87. doi: 10.1126/science.167.3914.86

Gallup, G. G. (1977). Absence of self-recognition in a monkey (Macaca fascicularis) following prolonged exposure to a mirror. Dev. Psychobiol. 10, 281-284. doi: 10.1002/dev.420100312

Goodall, J. (1986). The Chimpanzees of Gombe: Patterns of Behavior. Cambridge: Harvard University Press.

Haun, D. B. M., and Call, J. (2008). Imitation recognition in great apes. Curr. Biol. 18, 288-290. doi: 10.1016/j.cub.2008.02.031

Hayes, K. J., and Hayes, C. (1952). Imitaiton in a home-rasied chimpanzee. J. Comp. Physiol. Psychol. 45, 450-459. doi: 10.1037/h0053609

Hecht, E. E., Gutman, D. A., Preuss, T. M., Sanchez, M. M., Parr, L. A., and Rilling, J. K. (2013). Process versus product in social learning: comparative diffusion tensor imaging of neural systems for action execution-observation matching in macaques, chimpanzees, and humans. Cereb. Cortex 23, 1014-1024. doi: 10.1093/cercor/bhs097

Herrmann, E., Call, J., Hernandez-Lloreda, M. V., Hare, B., and Tomasello, M. (2007). Humans have evolved specialized skills of social cognition: the cultural intelligence hypothesis. Science 317, 1360-1366. doi: 10.1126/science.1146282

Herrmann, E., Hare, B., Call, J., and Tomasello, M. (2010a). Differences in the cognitive skills of bonobos and chimpanzees. PLOS ONE 5:e12438. doi: 10.1371/journal.pone.0012438

Herrmann, E., Hernandez-Lloreda, M. V., Call, J., Hare, B., and Tomasello, M. (2010b). The structure of individual differences in the cognitive abilities of children and chimpanzees. Psychol. Sci. 21, 102-110. doi: 10.1177/0956797609 356511

Hobaiter, C., and Byrne, R. W. (2010). Able-bodied wild chimpanzees imitate a motor procedure used by a disabled individual to overcome a handicap. PLoS ONE 5:e11959. doi: 10.1371/journal.pone.0011959

Hobson, J. A., and Hobson, R. P. (2007). Identification: the missing link between joint attention and imitation? Dev. Psychopathol. 10, 411-431.

Hopkins, W. D., Donaldson, Z. R., and Young, L. Y. (2012). A polymorphic indel containing the RS3 microsatellitein the $5^{\prime}$ flanking region of the vasopressin V1a receptor gene is associated with chimpanzee (Pan troglodytes) personality. Genes, Brain and Behav. 11, 552-558. doi: 10.1111/j.1601-183X.2012. 00799.x 
Hopkins, W. D., Keebaugh, A. C., Reamer, L. A., Schaeffer, J., Schapiro, S. J., and Young, L. J. (2014a). Genetic influences on receptive joint attention in chimpanzees (Pan troglodytes). Sci. Rep. 4, 1-7. doi: 10.1038/srep 03774

Hopkins, W. D., Russell, J. L., and Schaeffer, J. (2014b). Chimpanzee intelligence is heritable. Curr. Biol. 24, 1649-1652. doi: 10.1016/j.cub.2014. 05.076

Hopkins, W. D., and Taglialatela, J. P. (2013). Initiation of joint attention is associated with morphometric variation in the anterior cingulate cortex of chimpanzees (Pan troglodytes). Am. J. Primatol. 75, 441-449. doi: 10.1002/ajp. 22120

Horner, V., and Whiten, A. (2005). Causal knowledge and imitation/emulation switching in chimpanzees (Pan troglodytes) and children (Homo sapiens). Anim. Cogn. 8, 164-181. doi: 10.1007/s10071-004-0239-6

Iacoboni, M. (2009). Imitation, empathy and mirror neurons. Annu. Rev. Psychol. 60, 653-670. doi: 10.1146/annurev.psych.60.110707.163604

Ingersoll, B., and Schreibman, L. (2006). Teaching reciprocal imitation skills to young children with autism using a naturalistic behavioral approach: effects on language, pretend play, and joint attention. J. Autism. Dev. Disord. 36, 487-505. doi: 10.1007/s10803-006-0089-y

Latzman, R. D., Hopkins, W. D., Keebaugh, A. C., and Young, L. J. (2014). Personality in chimpanzees (Pan troglodytes): exploring the hierarchical structure and associations with the aasopressin V1A receptorg gene. PLoS ONE 9:e95741. doi: 10.1371/journal.pone.0095741

Leavens, D. A. (2012). “Joint attention: twelve myths," in Joint Attention: New Developments in Pyschology, Philosophy of Mind and Social Neuroscience, ed. A. Seemann (Cambridge, MA: MIT Press), 43-72.

Leavens, D. A., and Hopkins, W. D. (1998). Intentional communication by chimpanzee (Pan troglodytes): a cross-sectional study of the use of referential gestures. Dev. Psychol. 34, 813-822. doi: 10.1037/0012-1649.34.5.813

Leavens, D. A., Hopkins, W. D., and Bard, K. A. (2008). "The heterochronic origin of explicit reference," in The Shared Mind: Perspective on Intersubjectivity, eds J. Zlatev, T. P. Racine, C. Sinha, and E. Itkonen (Amsterdam: John Benjamins), 187-214. doi: 10.1075/celcr.12.12lea

Leavens, D. A., and Racine, T. P. (2009). Joint attention in apes and humans: are humans unique? J. Consci. Stud. 16, 240-267.

Lin, A. C., Bard, K. A., and Anderson, J. R. (1992). Development of selfrecognition in chimpanzees (Pan troglodytes). J. Comp. Psychol. 106, 120-127. doi: 10.1037/0735-7036.106.2.120

Lyn, H., Russell, J. L., Leavens, D. A., Bard, K. A., Boysen, S. T., Schaeffer, J. A., et al. (2013). Apes communicate about absent and displaced objects: methodology matters. Anim. Cogn. 17, 85-94. doi: 10.1007/s10071-0130640-0

Meltzoff, A. N. (1990). "Foundations for developing a concept of self: the role of imitation in relating self to other and the value of social mirroring, social modelling, and the self practice in infancy," in The Self in Transition: Infancy to Childhood, eds D. Cicchetti and M. Beeghly (Chicago, IL: University of Chicago Press), 139-164.

Meltzoff, A. N. (2002). "Imitation as a mechanism of social cognition: Origins of empathy, theory of mind and representation of action," in Blackwell Handbook of Childhood Cognitive Development, ed. U. Goswami (Oxford: Blackweel Publishers).

Meltzoff, A. N., and Decety, J. (2003). What imitation tells us about social cognition: a rapprochement between developmental psychology and cognitive neuroscience. Philos. Trans. R. Soc. Lond. Ser. B Biol. Sci. 358, 491-500. doi: 10.1098/rstb.2002.1261

Meltzoff, A. N., and Prinz, W. (eds). (2002). The imitative mind: Development, Evolution and Brain Bases. Cambridge: Cambridge University Press. doi: 10.1017/CBO9780511489969

Mitani, J. C., Merriwether, D. A., and Zhang, C. (2000). Male affiliation, cooperation, and kinship in wild chimpanzees. Anim. Behav. 59, 885-893. doi: 10.1006/anbe.1999.1389

Mitchell, R. W. (1993). Mental models of mirror-self recognition: two theories. New Ideas Psychol. 11, 295-325. doi: 10.1016/0732-118X(93) 90002-U

Mitchell, R. W. (1997). A comparison of the self-awareness and kinesthetic matching theories of self-recognition: autistic children and others. Ann. N. Y. Acad. Sci. 818, 39-62. doi: 10.1111/j.1749-6632.1997.tb48245.x
Moore, B. R. (1992). Avian movement imitaiton and a new form of mimicry: traching the evolution of a complex form of learning. Behaviour 122, 231-263. doi: $10.1163 / 156853992 X 00525$

Myowa-Yamakoshi, M., and Matsuzawa, T. (1999). Factors influencing imitation of manipulatory actions in chimpanzees (Pan troglodytes). J. Comp. Psychol. 113, 128-136. doi: 10.1037/0735-7036.113.2.128

Myowa-Yamakoshi, M., Tomonaga, M., Tanaka, M., and Matsuzawa, T. (2004). Imitaiton in neonatal chimpanzees (Pan troglodytes). Dev. Sci. 7, 437-442. doi: 10.1111/j.1467-7687.2004.00364.x

Nadel, J. (2002). "Imitation and imitation recognition: Their functional role in preverbal infants and nonverbal children with autism," in The Imitative Mind: Development, Evolution and Brain Bases, eds A. Meltzoff and W. Prinz (Cambridge, MA: Cambridge University Press), 42-62.

Nielsen, M., Collier-Baker, E., Davis, J. M., and Suddendorf, T. (2005). Imitation recognition in a captive chimpanzee (Pan troglodytes). Anim. Cogn. 8, 31-36. doi: 10.1007/s10071-004-0232-0

Nielsen, M., and Dissanayake, C. (2004). Pretend play, mirror self-recognition and imitation: a longitudinal investigation through the second year. Infant Behav. Dev. 27, 342-365. doi: 10.1016/j.infbeh.2003.12.006

Nielsen, M., Suddendorf, T., and Dissanayake, C. (2006). "Imitation and selfrecognition in autism: in search of an explanation," in Imitation and the Social Mind, eds S. J. Rogers and J. H. G. Williams (New York: Guilford Press).

Paukner, A., Anderson, J. R., Borelli, E., Visalberghi, E., and Ferrari, P. F. (2005). Macaques (Macaca nemistrina) recognize when they are being imitated. Biol. Lett. 1,219-222. doi: 10.1098/rsbl.2004.0291

Paukner, A., Suomi, S. J., Visalberghi, E., and Ferrari, P. F. (2009). Capuchin monkeys display affiliation toward humans who imitate them. Science 325, 880-883. doi: 10.1126/science. 1176269

Povinelli, D. J., Gallup, G. G., Eddy, T. J., Bierschwale, D. T., Enstrom, M. C., Perilloux, H. K., et al. (1997). Chimpanzees recognize themselves in mirrors. Anim. Behav. 53, 1083-1088. doi: 10.1006/anbe.1996.0303

Rizzolatti, G., and Craighero, L. (2004). The mirror-neuron system. Annu. Rev. Neurosci. 27, 169-192. doi: 10.1146/annurev.neuro.27.070203.144230

Rizzolatti, G., and Luppino, G. (2001). The cortical motor system. Neuron 31, 889-901. doi: 10.1016/S0896-6273(01)00423-8

Rogers, S. J., Hepburn, S. L., Stackhouse, T., and Wehner, E. (2003). Imitation performance in toddlers with autism and those with other developmental disorders. J. Child Psychol. Psychiatry 44, 763-781. doi: 10.1111/1469-7610. 00162

Russell, J. L., Lyn, H., Schaeffer, J. A., and Hopkins, W. D. (2011). The role of sociocommunicative rearing environments in the development of social and physical cognition in apes. Dev. Sci. 14, 1459-1470. doi: 10.1111/j.1467-7687.2011. 01090.x

Schmitt, V., Pankau, B., and Fischer, J. (2011). Old world monkeys compare to apes in the primate cognition test battery. PLOS ONE 7:e32024. doi: 10.1371/journal.pone.0032024

Sclafani, V., Paukner, A., Suomi, S. J., and Ferrari, P. F. (2014). Imitation promotes affiliation in infant macaques at risk for impaired social behaviors. Dev. sci. doi: 10.1111/desc.12237 [Epub ahead of print].

Slaughter, V., and McConnell, D. (2003). Emergence of joint attention: relationships between gaze following, social referencing, imitation and naming in infancy. J. Genet. Psychol. 164, 54-71. doi: 10.1080/002213203095 97503

Sowden, S., and Shah, P. (2014). Self-other control: a candidate mechanism for social cognitive function. Front. Hum. Neurosci. 8:789. doi: 10.3389/fnhum.2014 00789

Tennie, C., Call, J., and Tomasello, M. (2006). Push or pull: imitation vs emulation in great apes and human children. Ethology 112, 1159-1169. doi: 10.1111/j.14390310.2006.01269.x

Thorndike, E. L. (1898). Animal intelligence: an experimental study of the associative processes in animals. Psychol. Rev. Monogr. 2, 551-553. doi: 10.1037/h0092987

Tomasello, M., Davis-Dasilva, M., Camak, L., and Bard, K. (1987). Observational learning of tool use by young chimpanzees. Hum. Evol. 2, 175-183. doi: 10.1007/BF02436405

Tomasello, M., Savage-Rumbaugh, S., and Kruger, A. C. (1993). Imitative learning of actions on objects by children, chimpanzees, and enculturated chimpanzees. Child Dev. 64, 1688-1705. doi: 10.2307/1131463 
Topal, J., Byrne, R. W., Miklosi, A., and Csanyi, V. (2006). Reproducing human actions and action sequences: "Do as I Do!" in a dog. Anim. Cogn. 9, 355-367. doi: 10.1007/s10071-006-0051-6

Uddin, L. Q., Iacoboni, M., Lange, C., and Keenan, J. P. (2007). The self and social cognition: the role of cortical midline structures and mirror neurons. Trends Cogn. Sci. 11, 153-157. doi: 10.1016/j.tics.2007. 01.001

Uddin, L. Q., Kaplan, J. T., Molnar-Szakacs, I., Zaidel, E., and Iacoboni, M. (2005). Self-face recognition activates a frontoparietal "mirror" network in the right hemisphere: an event-related fMRI study. Neuroimage 25, 926-935. doi: 10.1016/j.neuroimage.2004.12.018

Voelkl, B., and Huber, L. (2000). True imitation in marmosets. Anim. Behav. 60, 195-202. doi: 10.1006/anbe.2000.1457

Whalen, C., Schreibman, L., and Ingersoll, B. (2006). The collateral effect of joint attention training on social initiations, positive affect, imitation, and spontaneous speech in young children with autism. J. Autism. Dev. Disord. 36, 655-664. doi: 10.1007/s10803-0060108-z

Wrangham, R. W. (1986). "Ecology and social relationships in two species of chimpanzee," in Ecological Aspects of Social Evolution: Birds and Mammals, eds D. I. Rubenstein and R. W. Wrangham (Princeton, NJ: Princeton University Press), 352-378.

Conflict of Interest Statement: The authors declare that the research was conducted in the absence of any commercial or financial relationships that could be construed as a potential conflict of interest.

Received: 04 November 2014; accepted: 05 February 2015; published online: 26 February 2015.

Citation: Pope SM, Russell JL and Hopkins WD (2015) The association between imitation recognition and socio-communicative competencies in chimpanzees (Pan troglodytes). Front. Psychol. 6:188. doi: 10.3389/fpsyg.2015.00188

This article was submitted to Comparative Psychology, a section of the journal Frontiers in Psychology.

Copyright (c) 2015 Pope, Russell and Hopkins. This is an open-access article distributed under the terms of the Creative Commons Attribution License (CC BY). The use, distribution or reproduction in other forums is permitted, provided the original author(s) or licensor are credited and that the original publication in this journal is cited, in accordance with accepted academic practice. No use, distribution or reproduction is permitted which does not comply with these terms. 